\title{
Central Exclusive Production at the Tevatron
}

\author{
Michael G. Albrow \\ on behalf of the CDF Collaboration. \\ Fermi National Accelerator Laboratory, \\ P.O.Box 500, Wilson Road, Batavia, IL 60510, USA
}

DOI: http://dx.doi.org/10.3204/DESY-PROC-2009-01/22

\begin{abstract}
In CDF we have observed several exclusive processes: $\gamma \gamma \rightarrow e^{+} e^{-}$ and $\mu^{+} \mu^{-}, \gamma+\mathbb{P} \rightarrow J / \psi, \psi(2 S)$, and $\mathbb{P}+\mathbb{P} \rightarrow \chi_{c}$. The cross sections agree with QED, HERA photoproduction data, and theoretical estimates of $g g \rightarrow \chi_{c}$ with another gluon exchanged to screen the color. This observation of exclusive $\chi_{c}$, together with earlier observations of exclusive dijets and exclusive $\gamma \gamma$ candidates, support some theoretical predictions for $p+p \rightarrow p+H+p$ at the LHC. Exclusive dileptons offer the best means of precisely calibrating forward proton spectrometers.
\end{abstract}

\section{Central Exclusive Production}

Central exclusive production at the Tevatron is the process $p+\bar{p} \rightarrow p+X+\bar{p}$, where " + " means a rapidity gap $\Delta y$ exceeding 3 units, and $X$ is a simple system fully measured. Exchanges ( $t$-channel) over such large gaps must be color singlets with spin $J$ [or Regge intercept $\alpha(0)] \geq$ 1.0. Only photons $\gamma$ and pomerons $\mathbb{P}$ qualify, apart from $W$ and $Z$ bosons which always cause the proton to break up. The gluon $g$ would qualify apart from its color, but if another gluon is exchanged that can be cancelled, and $\mathbb{P}=g g$ is often a good approximation. It cannot be exact; QCD forbids a pure $g g$ state, and a $q \bar{q}$ component certainly grows as $Q^{2}$ increases. The $\mathbb{P}$ has $\mathrm{C}=+1$; in QCD one should also have a $g g g$ state with $\mathrm{C}=-1$, the odderon [1] $O$, not yet observed. The central masses $M_{X}$ are roughly limited to $M_{X} \lesssim \frac{\sqrt{s}}{20}$ with the outgoing protons having Feynman $x_{F}>0.95$. Hence $M_{X} \lesssim 3 \mathrm{GeV}$ at the CERN ISR [2], appropriate for glueball spectroscopy, where $M\left(\pi^{+} \pi^{-}\right)$shows a broad $f_{0}(600)$, a narrow $f_{0}(980)$ and still unexplained structure possibly associated with $f_{0}(1710)$, a glueball candidate. The study of $X=$ hadrons, e.g. $\phi \phi$ and $D^{\circ} \bar{D}^{\circ}$ to name two channels among many, has not been studied above ISR energies, but $\mathrm{CDF}$ is a perfect place to do it and hopefully it will be done.

At the LHC $M_{X}$ can reach $\approx 700 \mathrm{GeV}$, into the electroweak sector, and we can have $X=Z, H, W^{+} W^{-}, Z Z$, slepton pairs $\tilde{l} \tilde{l}$, etc. Measuring the forward protons after $120 \mathrm{~m}$ of 8T dipoles, in association with the central event, as the FP420 [4] proponents hope to do at ATLAS and CMS, one can measure $M_{X}$ with $\sigma\left(M_{X}\right) \approx 2 \mathrm{GeV}$ per event [5], and for a state such as $H$, also its width if $\Gamma(H) \gtrsim 3 \mathrm{GeV} / \mathrm{c}^{2}$. There are scenarios (e.g. SUSY) in which FP420 could provide unique measurements, e.g. if there are two nearby states both decaying to $b \bar{b}$ or to $W^{+} W^{-}$. The quantum numbers of $X$ are $J^{P C}=0^{++}$or $2^{++}$(and these are distinguishable) for $\mathbb{P} I P$ production. Two-photon collisions $\gamma \gamma \rightarrow l^{+} l^{-}, W^{+} W^{-}, \tilde{l} l$ become important at the LHC thanks to the intense high momentum photons, orders of magnitude more than at the Tevatron, 
giving $>50 \mathrm{fb}$ for $W^{+} W^{-}$as a continuum background to $H \rightarrow W^{+} W^{-} . H \rightarrow Z Z$ does not have this background.

While there is a gold mine of physics in $p+X+p$ at the LHC, we need to show that (a) the cross sections are within reach, and (b) one can build the spectrometers with resolution $\sigma\left(M_{X}\right) \approx$ $2 \mathrm{GeV} / \mathrm{c}^{2}$ and calibrate their momentum scale and resolution, to measure $\Gamma(H)$, and perhaps to distinguish nearby states. Both these issues are addressed by CDF in a "TeV4LHC" spirit, and they are also very interesting in their own right. The calculation of cross sections (e.g. [6]) involves, in addition to $\sigma(g g \rightarrow X)$, the unintegrated gluon distribution $g\left(x_{1}, x_{2}\right)$, rapidity gap survival probability (no other parton interactions), and the Sudakov factor (probability of no gluon radiation producing hadrons). The Durham group predicts $\sigma(S M H)$ for $p+H+p$ at the LHC $=3 \underset{\div 3}{\times 3} \mathrm{fb}$. At the Tevatron $p+H+\bar{p}$ is out of reach, but the process $p+\chi_{c}\left(\chi_{b}\right)+\bar{p}$ is identical as far as QCD is concerned, as is $p+\gamma \gamma+\bar{p}$. Measuring these constrains the $S M H$ cross section. In CDF we have looked for both exclusive $\gamma \gamma$ [7] and $\chi_{c}$ [8], without however having detectors able to see the $p$ and $\bar{p}$. Instead we added forward calorimeters $(3.5<|\eta|<5.1)$ and beam shower counters BSC $(5.5<|\eta|<7.4)$. If these are all empty there is a high probability that both $p$ and $\bar{p}$ escaped intact with small $|t|$. We also measured [9] exclusive dijets.

For the exclusive $\gamma \gamma$ search we triggered on events with two electromagnetic $(E M)$ clusters with $E_{T}>4 \mathrm{GeV}$ in the central calorimeter, with a veto on signals in the BSC. This killed pile-up events and enabled us to take data without prescaling the trigger. We required all other detectors to be consistent with only noise; then our effective luminosity is only about $10 \%$ of the delivered luminosity. We found [7] 3 events with exactly two back-to-back $E M$-showers (assumed to be photons) with $M(\gamma \gamma)>10 \mathrm{GeV} / \mathrm{c}^{2}$. From wire proportional chambers at the shower maximum we concluded that two were perfect $p+\bar{p} \rightarrow p+\gamma \gamma+\bar{p}$ candidates and one was also consistent with being a $p+\bar{p} \rightarrow p+\pi^{\circ} \pi^{\circ}+\bar{p}$ event. The Durham prediction [10] was $0.8_{\div 3}^{\times 3}$ events, clearly consistent. We have since accumulated more data, with a lower threshold, now being analysed.

With the above trigger we also found [11] $16 p+\bar{p} \rightarrow p+e^{+} e^{-}+\bar{p}$ events, with $M\left(e^{+} e^{-}\right)>10 \mathrm{GeV} / \mathrm{c}^{2}$ (up to $38 \mathrm{GeV} / \mathrm{c}^{2}$ ), the QED $\gamma \gamma \rightarrow e^{+} e^{-}$process [12]. Exclusive 2-photon processes had not previously been observed in hadron-hadron collisions; the cross section agrees with the precise theory prediction. This process has been suggested as a means of calibrating the LHC luminosity; then it must be done in the presence of pile-up, and one will need to know the acceptance etc. at the few \% level. More interesting for FP420 is that measurement of an exclusive lepton pair gives both forward proton momenta, with a precision dominated by the incoming beam momentum spread $\left(\frac{\delta p}{p} \approx 10^{-4}\right.$, or $\left.700 \mathrm{MeV}\right)$. One can do this with pile-up, selecting dileptons with no associated tracks on the $l^{+} l^{-}$vertex and $\Delta \phi \approx \pi$. One can also cut on $p_{T}\left(l^{+} l^{-}\right)$(correlated with $\Delta \phi$ ), but $\Delta \phi$ has better resolution. In CDF we found that a cut $\pi-\Delta \phi<\frac{0.8 \mathrm{GeV}}{M\left(l^{+} l^{-}\right)}$rads is suitable for QED-produced pairs. For each pair one can predict $\xi_{1}$ and $\xi_{2}$, and, if a proton is in the FP420 acceptance, compare $\xi_{i}$ and $\xi_{420}$. This can also possibly map the acceptance $\mathrm{A}(\xi, t \approx 0)$, as the cross section shape is known from $\mathrm{QED}$, and the (Coulomb) protons have very small $t$.

CDF also used a "muon+track" trigger, again with BSC veto, to study $p+\bar{p} \rightarrow p+$ $\mu^{+} \mu^{-}+\bar{p}$ with $3 \mathrm{GeV} / \mathrm{c}^{2}<M(\mu \mu)<4 \mathrm{GeV} / \mathrm{c}^{2}$. This is a very rich region, with the $J / \psi$ and $\psi(2 S)$ vector mesons that can only be produced exclusively by photoproduction $\gamma+\mathbb{P} \rightarrow \psi$, or 


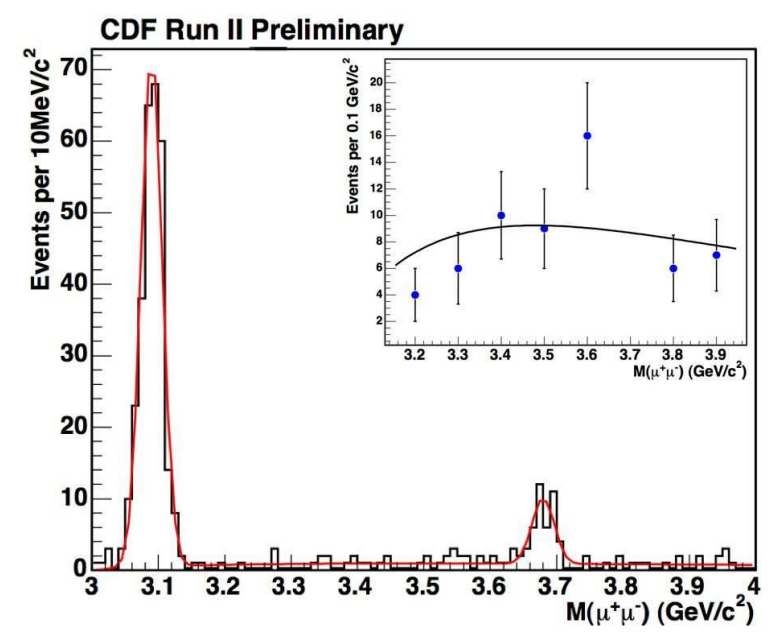

Fig. 1: Exclusive dimuon mass spectrum in the charmonium region, together with the sum of two Gaussians and the QED continuum, shown in the inset, excluding the $3.65-3.75 \mathrm{GeV} / \mathrm{c}^{2}$ bin $(\psi(2 S))$. All line shapes are predetermined, with the normalization free.

possibly by odderon exchange: $O+\mathbb{P} \rightarrow \psi$. We know what to expect for photoproduction from HERA, so an excess would be evidence for the elusive $O$. The spectrum [8] is shown in Fig. 1, together with the sum of three components: the vector mesons and a continuum, $\gamma \gamma \rightarrow \mu^{+} \mu^{-}$, which is again consistent with QED. These central exclusive spectra are exceptionally clean; in fact the biggest background $(\approx 10 \%)$ is the identical process but with an undetected $p \rightarrow p^{*}$ dissociation. The $J / \psi$ and $\psi(2 S)$ cross sections $\left.\frac{d \sigma}{d y}\right|_{y=0}$, are (3.92 \pm 0.62$)$ nb and $(0.54 \pm 0.15) \mathrm{nb}$, agreeing with expectations $[13,14]$. Thus we do not have evidence for $O$ exchange, and put a limit $\frac{O}{\gamma}<0.34$ (95\% c.1.), compared with a theory prediction [15] $0.3-0.6$.

While the QED and photoproduction processes in Fig. 1 should hold no surprises, their agreement with expectations validates the analysis. We required no $E M$ tower with $E_{T}^{E M}>$ $80 \mathrm{MeV}$. If we allow such signals (essentially $\gamma$ 's) the number of $J / \psi$ events jumps from 286 to 352 , while the number of $\psi(2 S)$ only increases from 39 to 40 . The spectrum of EM showers is shown in Fig. 2. These extra $J / \psi$ events are very consistent with being $\chi_{c 0}(3415) \rightarrow J / \psi+\gamma$, from $\mathbb{P} \mathbb{P} \rightarrow \chi_{c}$, with about $20 \%$ of the $\gamma$ being not detected (giving a background of $4 \%$ under the exclusive $J / \psi)$. We measure $\left.\frac{d \sigma}{d y}\left(\chi_{c}\right)\right|_{y=0}=(75 \pm 14) \mathrm{nb}$. The existence of this process implies that $p+H+p$ must happen at the LHC (assuming $H$ exists), as the QCD physics is qualitatively identical. The $\chi_{c}$ cross section agrees with predictions: $150 \mathrm{nb}$ [16] and $130_{\div 4}^{\times 4} \mathrm{nb}$ [6]. It is therefore likely that $\sigma(p+p \rightarrow p+S M H+p)$ is of order 0.5-5 fb, within reach of FP420. In SUSY models the cross section can be much higher [4].

We are looking for $p+\bar{p} \rightarrow p+\Upsilon+\bar{p}$ (by photoproduction, or by $O+\mathbb{P}$ ), and $\mathbb{P}+\mathbb{P} \rightarrow \chi_{b}$. The $\Upsilon$ should be measurable in the presence of pile-up using $n_{\text {ass }}=0, \Delta \phi$ and $p_{T}$ cuts $\left(n_{\text {ass }}\right.$ is the number of additional tracks on the dilepton vertex). We have candidate events, with the $\Upsilon(1 S),(2 S)$ and $(3 S)$ states resolved; cross sections are now being determined. The $\chi_{b} \rightarrow \Upsilon+\gamma$ 


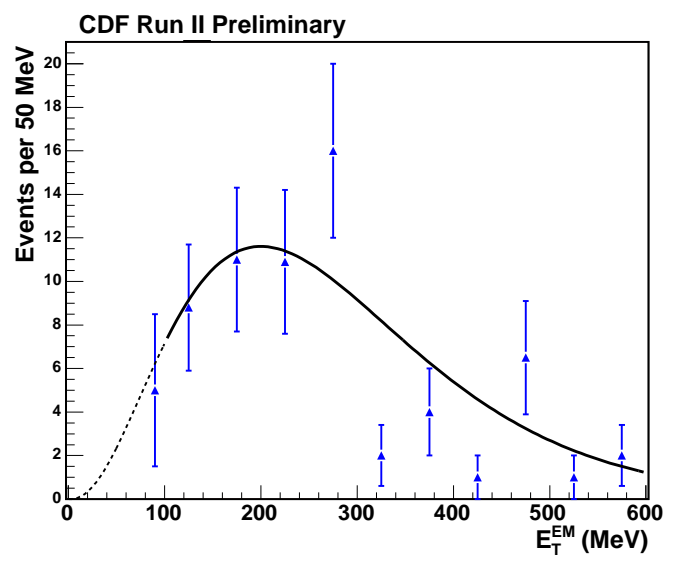

Fig. 2: The $E_{T}$ spectrum of electromagnetic showers associated with $J / \psi$, together with an empirical function to estimate the fraction under the $80 \mathrm{MeV}$ cut. These are $\chi_{c 0}(3415)$ candidates.

probably can not be studied in the presence of pile-up, and it is challenging. We have also made a search [17] for exclusive $Z$, allowed only through photoproduction: $\gamma+\mathbb{P} \rightarrow Z$. In the Standard Model the (integrated) cross section at the Tevatron is too small to see, $\sigma_{\text {excl }}(Z)=$ $0.3 \mathrm{fb}$ [14] or 1.3fb [18], before branching fractions. In White's pomeron theory [19] the cross section is expected to be much larger, but a quantitative prediction is lacking. Our search uses both $e^{+} e^{-}$and $\mu^{+} \mu^{-}$pairs with $M\left(l^{+} l^{-}\right)>40 \mathrm{GeV} / \mathrm{c}^{2}$. There are 8 exclusive candidates with $\sigma\left(p+\bar{p} \rightarrow p+\left(\gamma \gamma \rightarrow l^{+} l^{-}\right)+\bar{p}\right)=0.24_{-0.10}^{+0.13} \mathrm{pb}$ (for $|\eta(\mu)|<4.0$ ), agreeing with $\sigma(\mathrm{QED})$ $=0.256 \mathrm{pb}$. All the events have $\pi-\Delta \phi<0.013(\mathrm{rad})$ and $p_{T}\left(\mu^{+} \mu^{-}\right)<1.2 \mathrm{GeV} / \mathrm{c}$. Only one event had a $\bar{p}$ in the acceptance of the Roman pots when they were operational, and a track was observed, showing that the event was exclusive, and that at the LHC such $l^{+} l^{-}+p$ events will be available for calibration. If we remove the requirement that the BSC should be empty there are 4 additional events, interpreted as $p \rightarrow p^{*}$ dissocation. One of them has $M\left(\mu^{+} \mu^{-}\right) \approx M(Z)$ and a larger $\Delta \phi$ and $p_{T}$ than the others, but we cannot claim it to be truly exclusive. We put a limit on exclusive $\sigma_{\text {excl }}(Z)<0.96 \mathrm{pb}$ at $95 \%$ c.l. Clearly it will be interesting to look for exclusive $p+Z+p$ at the LHC. In early running of the LHC, when bunch crossings without pile-up are not yet rare, it is important to measure these exclusive processes, to the extent possible without complete forward coverage. In CMS we have plans to add forward shower counters [20] around the beam pipe to help tag rapidity gaps, together with the ZDC and forward hadron calorimeters. With large forward gaps in both directions, a trigger on two EM showers with $E_{T}>4 \mathrm{GeV}$ should be possible, hopefully observing $\Upsilon \rightarrow e^{+} e^{-}, \gamma \gamma \rightarrow e^{+} e^{-}, \mathbb{P} \mathbb{P} \rightarrow \gamma \gamma$, and $\chi_{b} \rightarrow \Upsilon+\gamma \rightarrow e^{+} e^{-} \gamma$. Clean single interactions are surely needed for the $\chi_{b}$ and $\mathbb{P} \mathbb{P} \rightarrow \gamma \gamma$; both channels are excellent tests of $p+H+p$. One may even hope that when exclusive Higgs production is measured, the coupling $g g H$ can be derived by comparing the three cross sections!

\section{References}

[1] See e.g. C.Ewerz, The odderon in Quantum Chromodynamics, hep-ph/0306137 (2003), and references therein. 
[2] T.Akesson et al. (AFS Collaboration), Nucl.Phys. B264, 154 (1986).

[3] At this meeting such a study was initiated by the Kiev group (V.Aushev, L.Jenkovszky et al.).

[4] M.G.Albrow et al., The FP420 R\&D project, Higgs and new physics with forward protons at the LHC, arXiv:0806.0302 [hep-ex].

[5] M.G.Albrow and A.Rostovtsev, Searching for the Higgs at hadron colliders using the missing mass method, hep-ph/0009336.

[6] V.A.Khoze et al., Eur.Phys.J. C35, 211 (2004); V.A.Khoze, A.D.Martin and M.G.Ryskin, Eur.Phys.J. C14, 525 (2000); A.De Roeck et al., Eur.Phys.J. C25, 391 (2002).

[7] A.Abulencia et al., (CDF Collaboration), Phys.Rev.Lett. 99, 242001 (2007).

[8] T.Aaltonen et al., (CDF Collaboration) Observation of exclusive charmonium production and $\gamma \gamma \rightarrow \mu^{+} \mu^{-}$in $p \bar{p}$ collisions at $\sqrt{s}=1.96 \mathrm{TeV}$; paper in preparation.

[9] T.Aaltonen et al., (CDF Collaboration) Phys.Rev. D77, 052004 (2008).

[10] V.A.Khoze et al., Eur.Phys.J. C38, 475 (2005).

[11] A.Abulencia et al. (CDF Collaboration), Phys.Rev.Lett. 98, 112001 (2007).

[12] J.Vermaseren, LPAIR, Nucl.Phys. B229, 347 (1983).

[13] E.g. S.Klein and J.Nystrand, Phys.Rev.Lett. 92, 142003 (2004).

[14] L.Motyka and G.Watt, Phys.Rev. D78, 014023 (2008).

[15] A.Bzdak et al., hep-ph/07021354 (2007).

[16] F.Yuan, Phys.Lett. B510, 155 (2001).

[17] CDF Collaboration, Search for exclusive $Z$ boson production; paper in preparation.

[18] V.P.Goncalves and M.V.T.Machado, Eur.Phys.J. C53, 33 (2008)

[19] A.R.White, Phys.Rev. D72, 036007 (2005). White does not claim that photoproduced $Z$ have to be exclusive.

[20] M.Albrow et al., Forward physics with rapidity gaps at the LHC, arXiv:0811:0120[hep-ex]. 